\title{
Meta-analysis of risk factors associated with oxaliplatin hypersensitivity reactions in cancer patients
}

\author{
Linhui Zhu ${ }^{1,2} \cdot$ Huan $\mathrm{Li}^{1,2} \cdot$ Qiong $\mathrm{Du}^{1,2} \cdot \mathrm{Xuan}_{\mathrm{Ye}^{1,2}} \cdot$ Sijia $\mathrm{Yu}^{1,2} \cdot \mathrm{Xin} \mathrm{Luo}^{1,2} \cdot$ Qing Zhai $^{1,2}$
}

Received: 26 May 2021 / Accepted: 15 September 2021 / Published online: 9 October 2021

(c) The Author(s) 2021

\begin{abstract}
This study aimed to investigate risk factors associated with oxaliplatin hypersensitivity reactions in cancer patients through a meta-analysis. A comprehensive retrieve of Chinese databases China National Knowledge Infrastructure, Wanfang Data, VIP Database and English databases PubMed, ScienceDirect, Embase and Cochrane library was conducted. The studies that meet the requirements for meta-analysis according to inclusion and exclusion criteria were screened and assessed for eligibility. Odds ratio (OR) / Weighted mean difference (WMD) and 95\% confidence intervals (95\% CIs) or calculable dichotomous and continuous raw data were extracted to perform meta-analysis using random effect model or fixed effect model on the basis of heterogeneity between studies through Review Manager 5.4 software. A total of 14 cross-sectional studies and 3367 cancer patients were included. Meta-analysis results showed that platinum exposure history (OR value 3.13, 95\% CI 2.19-4.48, heterogeneity $P=0.26$ ), allergy history (OR value $1.76,95 \% \mathrm{CI} 1.09-2.85$, heterogeneity $P=0.61$ ), platinum free interval (OR value $3.75,95 \%$ CI $2.00-7.06$, heterogeneity $P=0.83$ ), dexamethasone premedication dose (OR value 0.28 , $95 \%$ CI $0.13-0.58$, heterogeneity $P=0.21$ ) were significantly correlated to oxaliplatin hypersensitivity reactions. Gender, age, metastasis, combination with bevacizumab, XELOX regimen and cancer types were detected to have no statistically significant effect on oxaliplatin hypersensitivity reactions. Platinum exposure history, allergy history and long platinum-free interval are risk factors of oxaliplatin hypersensitivity reactions. High dexamethasone premedication dose is a protective factor of oxaliplatin hypersensitivity reactions.
\end{abstract}

Keywords Oxaliplatin $\cdot$ Hypersensitivity reactions $\cdot$ Risk factors $\cdot$ Meta-analysis

\section{Introduction}

Cancer has been a public health problem and the main cause of death in patients worldwide. In 2018 there were about 18 million new cancer cases worldwide and 9.6 million patients died [1], which had seriously affected human life quality. Platinum drugs, as the basic drugs in chemotherapy for cancer patients, are widely used in the treatment of a variety of malignant tumors. Oxaliplatin is the thirdgeneration platinum cytotoxic compound, which contains

Linhui Zhu and Huan Li contributed equally to this work.

Qing Zhai

zhaiqing63@126.com

1 Department of Pharmacy, Fudan University Shanghai Cancer Center, Shangai 200032, China

2 Department of Oncology, Shanghai Medical College, Fudan University, Shanghai 200032, China a 1,2 diaminocyclohexane carrier and an oxalate-based ligand. As a broad-spectrum anti-tumor agent, oxaliplatin has been clinically used in the treatment of multiple cancers [2]. Oxaliplatin adverse reactions are mainly manifested in neurotoxicity, hematological toxicity and gastrointestinal toxicity, and apart from these reactions oxaliplatin may also cause hypersensitivity reactions in cancer patients [3]. Hypersensitivity reaction is defined as an unexpected reaction with signs and symptoms not consistent with known toxicity of the drug [4]. Common clinical manifestations of oxaliplatin hypersensitivity reactions are pruritus, skin rash, dizziness, diarrhea, bronchospasm, itching, blood pressure changes, etc. and moreover, severe reactions may even lead to death events [3]. In recent years as oxaliplatin application in adjuvant and palliative chemotherapy has increased, the incidence of hypersensitivity reactions has further risen up between 8.9 and $24.0 \%$ [5-9]. For some cancer patients, oxaliplatin has become a vital part of the chemotherapy regimen. If a severe hypersensitivity reaction occurs which 
interrupts chemotherapy process and oxaliplatin reintroduction, disease control may be delayed and overall survival may also be shortened. Therefore, as an important and nonnegligible adverse reaction in clinical practice, investigating risk factors of oxaliplatin hypersensitivity reactions can help clinicians be aware of patients monitoring and clinical management. As current researches on risk factors of oxaliplatin hypersensitivity reactions have still not drawn unified conclusion, this study aims to systematically explore the association between possible risk factors and oxaliplatin hypersensitivity reactions by means of a meta-analysis.

\section{Materials and methods}

\section{Search strategy}

This meta-analysis was conducted on the basis of the meta-analysis of Observational Studies in Epidemiology (MOOSE) guidelines [10]. The literature search was conducted in Chinese databases China National Knowledge Infrastructure, Wanfang Data, VIP Database and foreign databases PubMed, ScienceDirect, Embase and Cochrane library, using combinations of the following key terms: (oxaliplatin) AND (hypersensitivity OR hypersensitivity reaction OR allergy OR allergic reaction) AND (risk factor). The full time period available for each database was searched up to February, 2021. Besides, references in searched relevant reviews were chosen for additional studies.

\section{Study selection}

Eligible studies needed to meet the following inclusion criteria: (1) the study population was cancer patients; (2) the study contained risk factors of oxaliplatin hypersensitivity reactions; (3) the study design was observational study; (4) the study reported odds ratio (OR) / weighted mean difference (WMD) with $95 \%$ confidence interval (CI) or raw data which can calculate these values; (5) oxaliplatin hypersensitivity reactions needed to be graded according to severity; (6) the study published language was Chinese or English. Exclusion criteria were the following: (1) review or comment; (2) case report; (3) abstract without full text; (4) the study with incomplete or incorrect data; (5) repetitive study or same population; (6) the study did not contain outcome related with risk factors of oxaliplatin hypersensitivity.

\section{Data extraction and quality assessment}

The following data were extracted from each study: (1) the first author's name and year of publication; (2) country; (3) study design; (4) study population; (5) number of cancer patients; (5) mean age of population; (6) number of men; (7) chemotherapy drugs; (8) median cycle; (9) median oxaliplatin cumulative dose; (10) involved risk factors; (11) incidence of oxaliplatin hypersensitivity reactions; (12) study quality score. OR/WMD value and $95 \%$ confidence interval with multivariate analysis would be directly extracted from each study (if the study did not conduct multivariate analysis, the results of univariate analysis would be accepted) or calculated based on the incidence of oxaliplatin hypersensitivity reactions in risk factors group and control group.

The Newcastle-Ottawa Quality Assessment Scale (NOS) was used to assess case-control studies and cohort studies [11]. The risk of bias was low if the score was 6 or more and the risk of bias was high if the score was 5 or less [12]. Agency for Healthcare Research and Quality (AHRQ) Cross-Sectional/Prevalence Study Quality was used to assess cross-sectional studies [11], with a score of 0-3 for lowquality study, $4-7$ for moderate-quality study and $8-11$ for high-quality study, respectively [13].

\section{Statistical analysis}

Review Manager 5.4 software was applied to conduct this meta-analysis of all included studies. The Q statistic test and $I^{2}$ value were calculated to assess heterogeneity between studies $[14,15] . P<0.1$ and $I^{2}>50 \%$ was considered high heterogeneity among studies and random effect model would be used to perform the analysis. $P \geq 0.1$ and $I^{2} \leq 50 \%$ was regarded low heterogeneity among studies and fixed effect model would be used to perform the analysis. Dichotomous outcomes were expressed as OR and 95\% CI and continuous outcome were expressed as WMD and 95\% CI with $P<0.05$ considered to be statistically significant. Forest plots were applied to display outcomes graphically and the funnel plot was used to examine the potential publication bias [16]. Sensitivity analyses were performed by applying different models on each factor and omitting one study at a time among high-heterogeneity risk factor group to assess influence on pooled estimate results.

\section{Results}

\section{Search results}

The literature search yielded a total of 141 potentially relevant articles, 35 of which were duplicate literatures. Sixtyfour studies were excluded due to irrelevant research topics with oxaliplatin hypersensitivity reactions through primary screening on title and abstract of each literature. Of the remains, 28 literatures not meeting inclusion criteria were removed out and 14 studies which contained investigation of oxaliplatin hypersensitivity reactions were included, 4 of which were published in Chinese from China National 
Knowledge Infrastructure, Wanfang Data and VIP Database. The flowchart of the process on literature search and study selection was shown in Fig. 1.

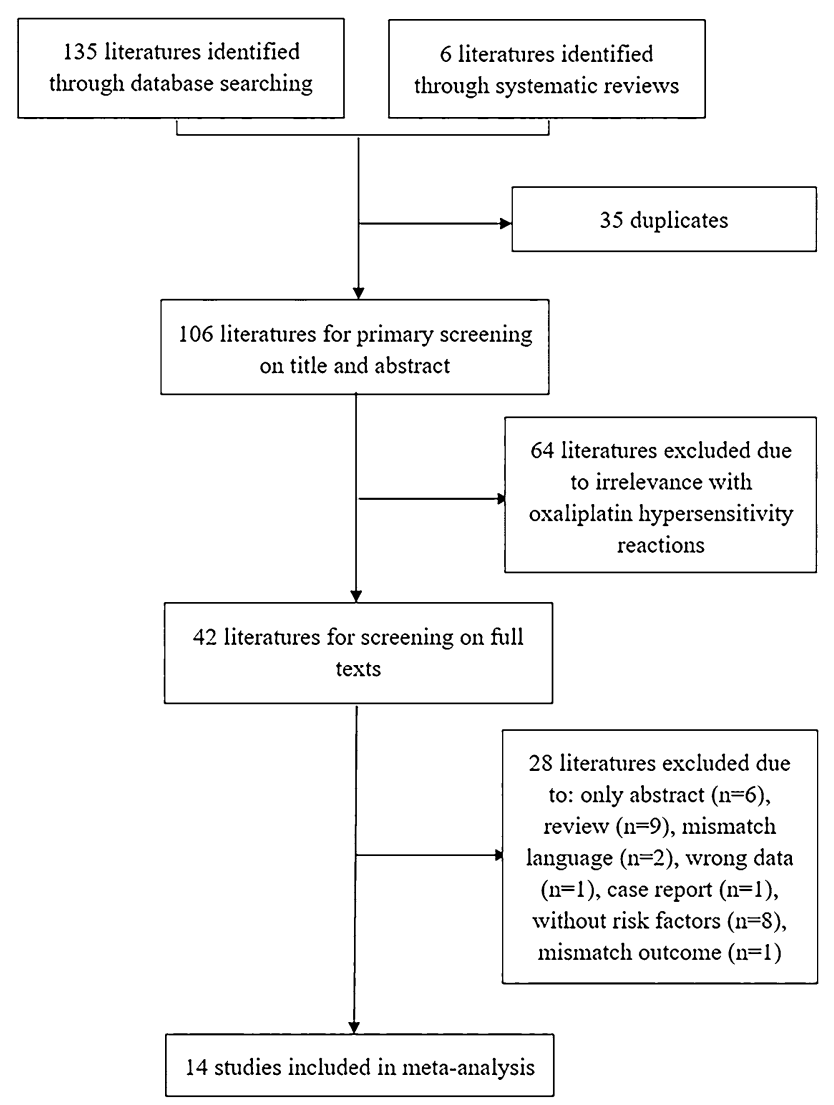

Fig. 1 Flowchart of study search and selection

\section{Study characteristics}

Table 1 reported basic characteristics of 14 studies [17-30] in this meta-analysis. All studies included for analysis were cross-sectional studies, which were published after 2009. The number of cancer patients ranged from 62 to 793 (total $N=3367$ ), with number of men patients from 35 to 481 and mean age from 51 to 66. Eight of the included studies were conducted in colorectal cancer patients, one was in gastrointestinal and urinary cancer patients and the other four were in all cancer patients with no restriction on cancer types. All studies were assessed for methodological quality with AHRQ Cross-Sectional/Prevalence Study Quality, 12 of which were moderate quality study and 2 of which were high-quality study. There were no studies with low-quality rating. Table 2 displays the outcome of oxaliplatin hypersensitivity reactions in each study. Cancer patients in studies received chemotherapy regimen containing oxaliplatin in combination with some of the following drugs: leucovorin, 5-FU, capecitabine, gemcitabine, $S$-1, docetaxel, etoposide, epirubicin, vindesine, raltitrexed, irinotecan, endostatin, bevacizumab, cetuximab, sunitinib and panitumumab. Oxaliplatin hypersensitivity reactions median chemotherapy cycle ranged from 5 to 9 with four studies no mention, while median cumulative oxaliplatin dose varied from 775.0 to $895.0 \mathrm{mg}$ and from 400.9 to $625.7 \mathrm{mg} / \mathrm{m}^{2}$ with seven studies no mention. Risk factors associated with oxaliplatin hypersensitivity reactions in each study included gender, age, platinum exposure history, allergy history, metastasis, platinum-free interval, combination with bevacizumab, XELOX regimen, dexamethasone premedication dose and cancer types. The incidence of oxaliplatin hypersensitivity

Table 1 Basic characteristics of included studies

\begin{tabular}{|c|c|c|c|c|c|c|c|}
\hline Author, year & Country & Study design & Study population & $\begin{array}{l}\text { Number of } \\
\text { patients }\end{array}$ & Mean age & Number of men & AHRQ score \\
\hline Li et al. 2018 [17] & China & Cross-sectional study & Colorectal cancer & 242 & 60 & 156 & 7 \\
\hline Du et al. 2014 [18] & China & Cross-sectional study & $\begin{array}{l}\text { Gastrointestinal } \\
\text { and urinary } \\
\text { cancer }\end{array}$ & 194 & 55 & 144 & 5 \\
\hline Shen et al. 2013[19] & China & Cross-sectional study & Colorectal cancer & 139 & 63 & 78 & 6 \\
\hline Zhu et al. 2017 [20] & China & Cross-sectional study & Colorectal cancer & 320 & 51 & 177 & 6 \\
\hline Ohta et al. 2017 [21] & Japan & Cross-sectional study & Colorectal cancer & 240 & 66 & 107 & 9 \\
\hline Mori et al. 2010 [22] & Japan & Cross-sectional study & Colorectal cancer & 223 & - & 148 & 5 \\
\hline Kim et al. 2009 [23] & America & Cross-sectional study & All cancer & 247 & 60 & 125 & 6 \\
\hline Okayama et al. 2015 [24] & Japan & Cross-sectional study & Colorectal cancer & 162 & 64 & 88 & 7 \\
\hline Parel et al. 2014 [25] & France & Cross-sectional study & All cancer & 119 & 62 & 78 & 7 \\
\hline Kim et al. 2012 [26] & Korea & Cross-sectional study & All cancer & 393 & 59 & 213 & 7 \\
\hline Sohn et al. 2018 [27] & Korea & Cross-sectional study & All cancer & 793 & 59 & 481 & 7 \\
\hline Shibata et al. 2009 [28] & Japan & Cross-sectional study & Colorectal cancer & 125 & 60 & 73 & 4 \\
\hline Yamauchi et al. 2015 [29] & Japan & Cross-sectional study & Colorectal cancer & 62 & 63 & 35 & 4 \\
\hline Seki et al. 2011 [30] & Japan & Cross-sectional study & Colorectal cancer & 108 & 64 & 67 & 8 \\
\hline
\end{tabular}


Table 2 Oxaliplatin hypersensitivity outcomes of each study

\begin{tabular}{|c|c|c|c|c|c|}
\hline Author, year & Chemotherapy drugs & $\begin{array}{l}\text { Median chemo- } \\
\text { therapy cycle }\end{array}$ & $\begin{array}{l}\text { Median cumulative } \\
\text { oxaliplatin dose }\end{array}$ & Risk factors & $\begin{array}{l}\text { Hypersensitivity } \\
\text { reactions inci- } \\
\text { dence/\% }\end{array}$ \\
\hline Li et al. 2018 [17] & abcdk & 6 & $895.0 \mathrm{mg}$ & (4)(6) 8 & 4.9 \\
\hline Du et al. 2014 [18] & abcdefghjlm & 7 & $478.7 \mathrm{mg} / \mathrm{m}^{2}$ & (1)(2)(4) & 10.8 \\
\hline Shen et al. 2013 [19] & abcdfl & 7 & $775.0 \mathrm{mg}$ & (1)(9) & 10.1 \\
\hline Zhu et al. 2017 [20] & abcdk & 8 & $625.7 \mathrm{mg} / \mathrm{m}^{2}$ & (1) (10) & 6.6 \\
\hline Ohta et al. 2017 [21] & $a b c$ & - & - & (3)(5)(7) & 16.3 \\
\hline Mori et al. 2010 [22] & $a b c$ & - & - & (1)(4)6(10) & 20.2 \\
\hline Kim et al. 2009 [23] & abcdek & 7 & - & (1)(2)(7)(10) & 11.7 \\
\hline Okayama et al. 2015 [24] & abcdkl & 8 & $582.0 \mathrm{mg} / \mathrm{m}^{2}$ & (1)(2)(5) 8 & 17.2 \\
\hline Parel et al. 2014 [25] & abcdeiklno & 5 & $400.9 \mathrm{mg} / \mathrm{m}^{2}$ & (1)(2)(3)(4) & 8.9 \\
\hline Kim MY et al. 2012 [26] & abcdkp & 8 & - & (1)(9) & 10.7 \\
\hline Sohn et al. 2018 [27] & abcdfo & - & - & (1)(3)6 & 18.7 \\
\hline Shibata et al. 2009[28] & $a b c$ & 9 & - & (1)(4) & 17.0 \\
\hline Yamauchi et al. 2015 [29] & abcdfkq & 8 & $544.5 \mathrm{mg} / \mathrm{m}^{2}$ & (1)(2)(3)(5)(7) & 11.3 \\
\hline Seki et al. 2011[30] & $a b c$ & - & - & (1)(4) & 22.2 \\
\hline
\end{tabular}

Chemotherapy drugs: $a=$ oxaliplatin; $b=$ leucovorin, $c=5-F U, d=$ capecitabine, e= gemcitabine, $f=S-1, g=$ docetaxel, $h=$ etoposide, $i=$ epirubicin, $\mathrm{j}=$ vindesine, $\mathrm{k}=$ bevacizumab, $\mathrm{l}=$ cetuximab, $\mathrm{m}=$ endostatin, $\mathrm{n}=$ raltitrexed, $\mathrm{o}=$ irinotecan, $\mathrm{p}=$ sunitinib $\mathrm{q}=$ panitumumab

Risk factors: (1) gender, (2) age, (3) platinum exposure history, (4) allergy history, (5)metastasis, (6) platinum free interval, (7) combination with bevacizumab, (8) XELOX regimen, (9) dexamethasone premedication dose, (10) cancer types

reactions remained between 4.9 and $22.2 \%$, among which two Chinese studies reported lower hypersensitivity reactions incidence (4.9 and 6.6\%) than current reviews $[17,20]$.

\section{Heterogeneity}

This meta-analysis investigated ten risk factors related to oxaliplatin hypersensitivity reactions. Among them, gender $\left(I^{2}=52 \%, P=0.02\right)$ and age $\left(I^{2}=82 \%, P=0.0002\right)$ were analyzed through random effect model. Other risk factors including platinum exposure history $\left(I^{2}=25 \%, P=0.26\right)$, allergy history $\left(I^{2}=0 \%, P=0.61\right)$, metastasis $\left(I^{2}=0 \%\right.$, $P=0.47)$, platinum-free interval $\left(I^{2}=0 \%, P=0.83\right)$, combination with bevacizumab $\left(I^{2}=0 \%, P=0.86\right)$, XELOX regimen $\left(I^{2}=7 \%, P=0.34\right)$, dexamethasone premedication dose $\left(I^{2}=35 \%, P=0.21\right)$ and cancer type (colon cancer) $\left(I^{2}=0 \%, P=0.46\right)$ were analyzed through fixed effect model.

\section{Risk factors associated with oxaliplatin hypersensitivity reactions}

\section{Gender}

Twelve studies were involved into analysis to compare patients in female group with those in male group and the pooled OR for oxaliplatin hypersensitivity reactions was 1.40 (95\% CI $0.89 \sim 2.21, Z=1.44, P=0.15$ ) to show no statistically significant differences between both groups. The forest plot was displayed in Fig. 2.

\section{Age}

Five studies were involved in the analysis to compare patients in hypersensitivity reaction group with those in non-hypersensitivity reaction group and the pooled WMD for age was $-1.93(95 \% \mathrm{CI}-6.09 \sim 2.22, Z=0.91, P=0.36)$ to show no statistically significant differences between both groups. The forest plot is displayed in Fig. 3.

\section{Platinum exposure history}

Four studies were involved in the analysis to compare patients in platinum exposure history group with those in no platinum exposure history group and the pooled OR for oxaliplatin hypersensitivity reactions was $3.13(95 \% \mathrm{CI}$ 2.19-4.48, $Z=6.25, P<0.00001)$ to show platinum exposure history considerably increased the risk of oxaliplatin hypersensitivity reactions. The forest plot is displayed in Fig. 4.

\section{Allergy history}

Six studies were involved in the analysis to compare patients in allergy history group with those in no allergy history group and the pooled OR for oxaliplatin hypersensitivity reactions was $1.76(95 \% \mathrm{CI} 1.09-2.85, Z=2.30, P=0.02)$ to show allergy history considerably increased the risk of 
Fig. 2 Forest plot of the association with gender and oxaliplatin hypersensitivity reactions
Fig. 3 Forest plot of the association with age and oxaliplatin hypersensitivity reactions

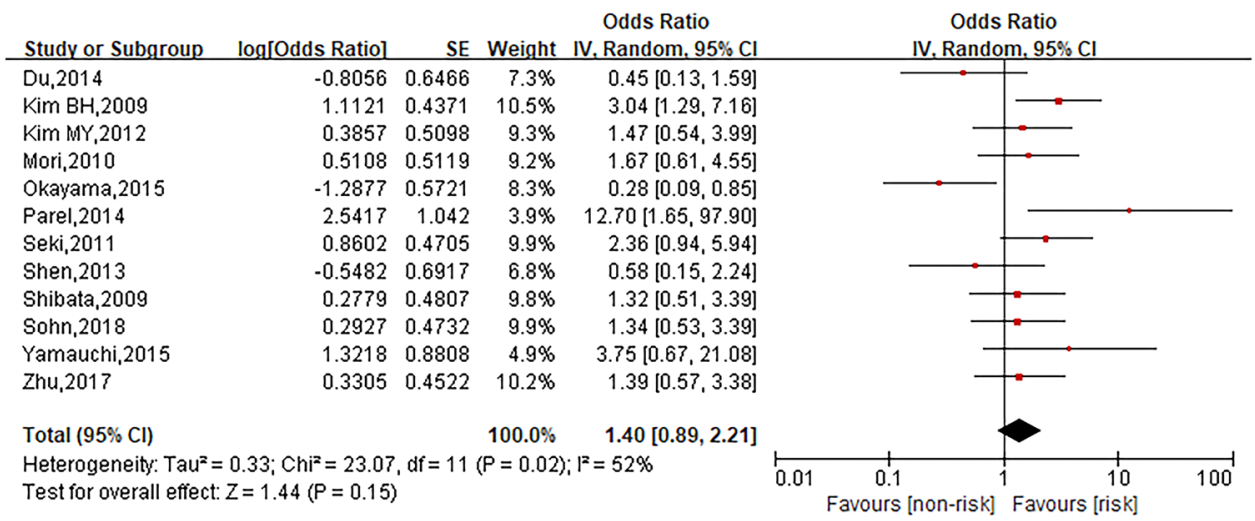

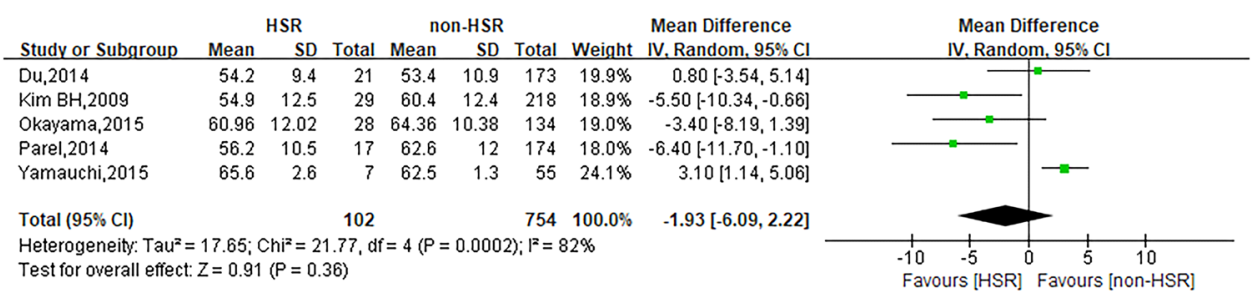

Fig. 4 Forest plot of the association with platinum exposure history and oxaliplatin hypersensitivity reactions

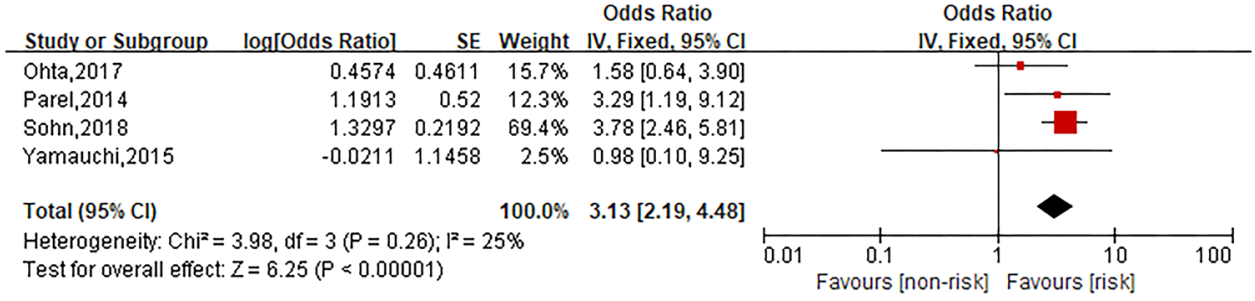

Fig. 5 Forest plot of the association with allergy history and oxaliplatin hypersensitivity reactions

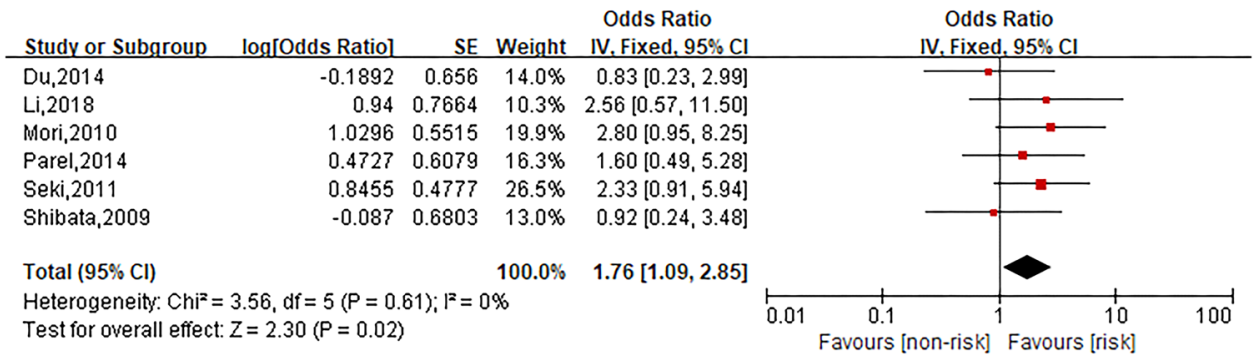

oxaliplatin hypersensitivity reactions. The forest plot is displayed in Fig. 5.

\section{Metastasis}

Three studies were involved in the analysis to compare patients in metastasis group with those in no metastasis group, and the pooled OR for oxaliplatin hypersensitivity reactions was 1.28 (95\% CI $0.58-2.82, Z=0.61, P=0.54)$ that showed no statistically significant differences between both groups. The forest plot is displayed in Fig. 6.

\section{Platinum-free interval}

Three studies were involved in the analysis to compare patients in long platinum free interval group with those in short platinum free interval group and the pooled OR for oxaliplatin hypersensitivity reactions was 3.75 (95\% CI 2.00-7.06, $Z=4.10, P<0.0001)$ that showed long platinum-free interval considerably increased the risk of oxaliplatin hypersensitivity reactions. The forest plot is displayed in Fig. 7. 

association with metastasis and oxaliplatin hypersensitivity reactions
Fig. 6 Forest plot of the

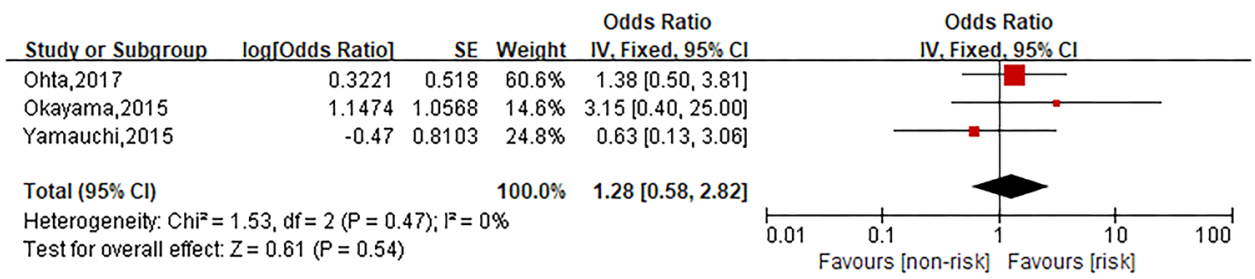

Fig. 7 Forest plot of the association with long platinum free interval and oxaliplatin hypersensitivity reactions

Fig. 8 Forest plot of the association with combination with bevacizumab and oxaliplatin hypersensitivity reactions

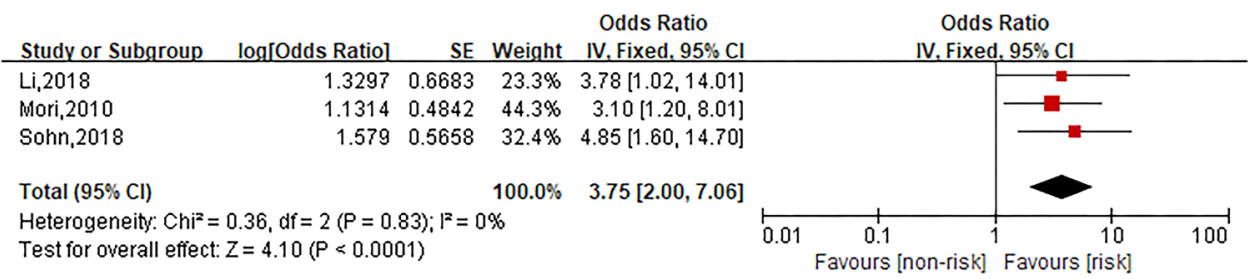

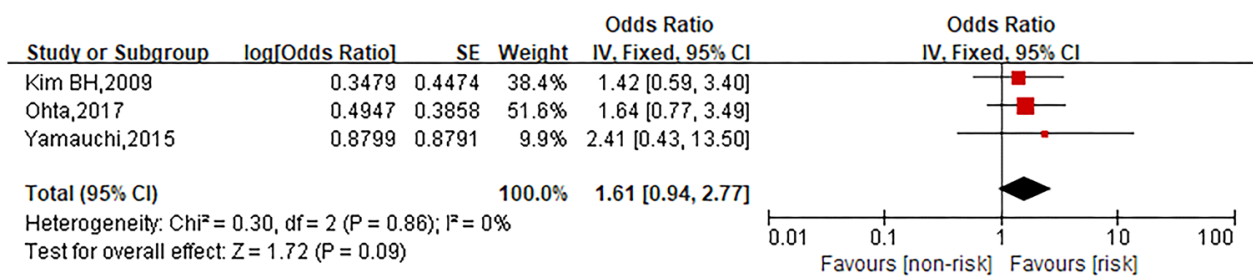

Fig. 9 Forest plot of the association with XELOX regimen and oxaliplatin hypersensitivity reactions

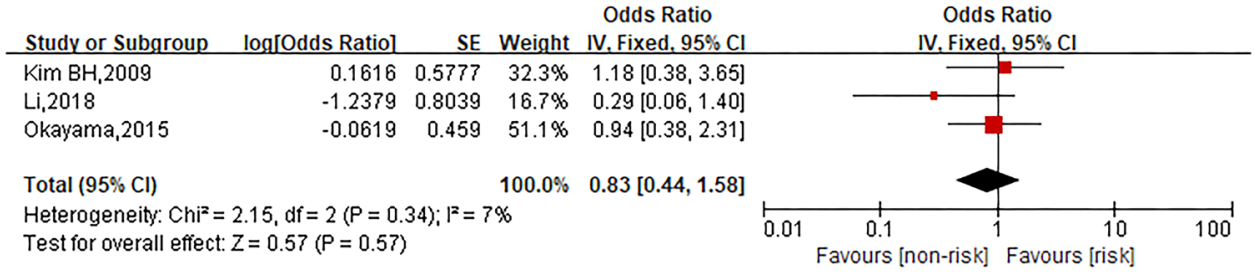

\section{Combination with bevacizumab}

Three studies were involved in the analysis to compare patients in bevacizumab group with those in no combination with bevacizumab group and the pooled OR for oxaliplatin hypersensitivity reactions was 1.61 (95\% CI 0.94-2.77, $Z=1.72, P=0.09)$ that showed no statistically significant differences between both groups. The forest plot is displayed in Fig. 8.

\section{XELOX regimen}

Three studies were involved in the analysis to compare patients in XELOX regimen group with those in other regimen group and the pooled OR for oxaliplatin hypersensitivity reactions was 0.83 (95\% CI $0.44-1.58, Z=0.57, P=0.57$ ) that showed no statistically significant differences between both groups. The forest plot is displayed in Fig. 9 .

\section{Dexamethasone premedication dose}

Three studies were involved in the analysis to compare patients in high dose group with those in low dose group and the pooled OR for oxaliplatin hypersensitivity reactions was 0.28 (95\% CI 0.13-0.58, $Z=3.39, P=0.0007$ ) to show high dexamethasone premedication dose considerably decreased the risk of oxaliplatin hypersensitivity reactions. The forest plot is displayed in Fig. 10.

\section{Cancer type (colon cancer)}

Three studies were involved in the analysis to compare patients in colon cancer group with those in other cancer types group and the pooled OR for oxaliplatin hypersensitivity reactions was 1.15 (95\% CI $0.69-1.90, Z=0.54, P=0.59)$ to show no statistically significant differences between both groups. The forest plot is displayed in Fig. 11. 
Fig. 10 Forest plot of the association with high dexamethasone premedication dose and oxaliplatin hypersensitivity reactions

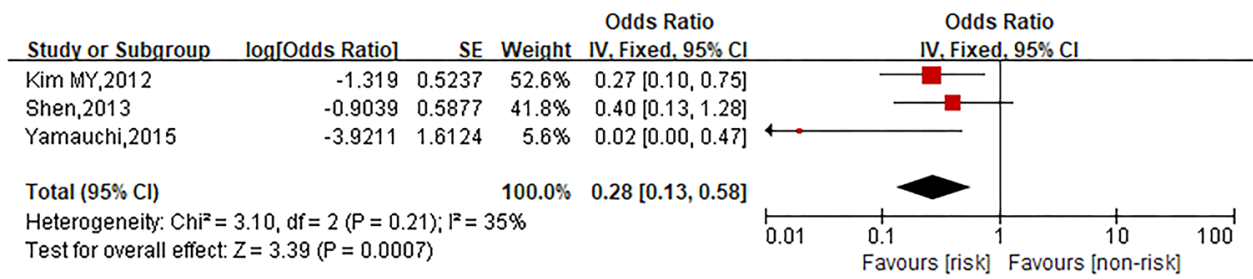

Fig. 11 Forest plot of the association with colon cancer and oxaliplatin hypersensitivity reactions

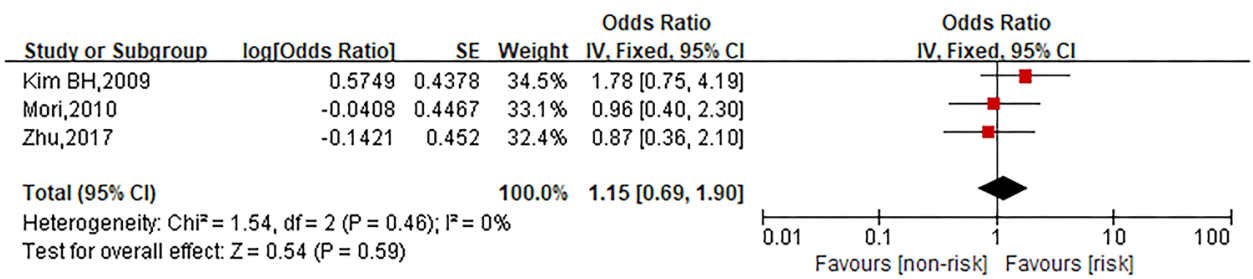

funnel plot in Fig. 12.

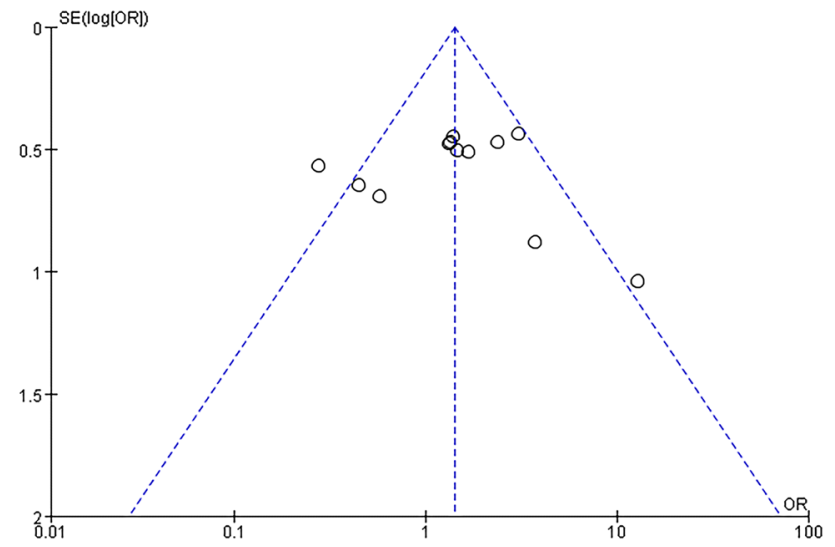

\section{Sensitivity analysis}

Another analysis model of each risk factor was used to perform analysis again and both results obtained from random effect model and fixed effect model were compared and exhibited in Table 3. Only conclusions drawn from two models of gender risk factor were different indicating obvious heterogeneity among studies, while other risk factors' results from two models were similar.

Risk factors with high heterogeneity of more than 50\% were eliminated studies one by one and pooled analysis again. (1) Gender: After omitting the study by Okayama et al., heterogeneity $I^{2}$ decreased from 52 to $30 \%$ and the result changed. The pooled OR value was 1.61 (95\% CI 1.09-2.38), suggesting that females may be more likely to develop oxaliplatin hypersensitivity reactions than males. (2) Age: After omitting the study by Yamauchi et al., heterogeneity $I^{2}$ decreased from 82 to $46 \%$ and the result changed. The pooled WMD value was -3.42 (95\% CI -6.69
Table 3 Pooled results of each risk factor with random effect model and fixed effect model

\begin{tabular}{lll}
\hline Risk factors & $\begin{array}{l}\text { Random effect model } \\
\text { OR/WMD [95\% CI }]\end{array}$ & $\begin{array}{l}\text { Fixed effect model } \\
\text { OR/WMD [95\% CI }]\end{array}$ \\
\hline Gender & $1.40[0.89,2.21]$ & $1.42[1.04,1.93]$ \\
Age & $-1.93[-6.09,2.22]$ & $0.55[-0.96,2.07]$ \\
Platinum exposure history & $2.85[1.74,4.66]$ & $3.13[2.19,4.48]$ \\
Allergy history & $1.76[1.09,2.85]$ & $1.76[1.09,2.85]$ \\
Metastasis & $1.28[0.58,2.82]$ & $1.28[0.58,2.82]$ \\
Platinum free interval & $3.75[2.00,7.06]$ & $3.75[2.00,7.06]$ \\
Combination with bevacizumab & $1.61[0.94,2.77]$ & $1.61[0.94,2.77]$ \\
XELOX regimen & $0.82[0.42,1.61]$ & $0.83[0.44,1.58]$ \\
Dexamethasone premedication dose & $0.25[0.09,0.69]$ & $0.28[0.13,0.58]$ \\
Cancer type (colon cancer) & $1.15[0.69,1.90]$ & $1.15[0.69,1.90]$ \\
\hline
\end{tabular}


to -0.16), suggesting that younger patients may be more likely to occur oxaliplatin hypersensitivity reactions than elder patients.

\section{Discussion}

Since oxaliplatin hypersensitivity reactions may interrupt basic chemotherapy of cancer patients, which puts adverse effects on disease control, it is crucial to identify risk factors of oxaliplatin hypersensitivity reactions early in clinical treatment to enhance patients' management. Although current studies have analyzed risk factors of oxaliplatin hypersensitivity reactions, the opinions are still not unified and no unanimous conclusion can be reached. On the basis of previous studies, this meta-analysis assessed the affection of gender, age, platinum exposure history, allergy history, metastasis, platinum-free interval, combination with bevacizumab, XELOX regimen, dexamethasone premedication dose and cancer types (colon cancer) on oxaliplatin hypersensitivity reactions, among which platinum exposure history, allergy history, platinum-free interval and dexamethasone premedication dose are typical features that may have representative significance.

The pathophysiology of oxaliplatin hypersensitivity reactions remains not clarified yet, but most widely accepted mechanism is generally explained with IgE-mediated type I hypersensitivity. Degranulation of mast cells and basophils leading to nonimmune-mediated histamine and cytokines released arouses capillary expansion and smooth muscle contraction [5-7]. Clinical manifestations (rash, bronchospasm, hypotension, etc. during or immediately after oxaliplatin infusion), occurring time (after multiple oxaliplatin chemotherapy cycles), skin test positive and platinum-specific IgE test positive all support such pathophysiology [31]. Some rare symptoms such as hemolysis, arthralgia, proteinuria, etc. may be related to type II and III hypersensitivity reactions, involving tissue deposition of drug-antibody complexes and activation of the complement pathway [5, 9]. IgE-mediated hypersensitivity reactions usually occur after exposure to allergen once [32], so patients with allergy history may have produced specific IgE in vivo. Although studies do not elucidate previous allergens of patients with oxaliplatin hypersensitivity reactions, according to a research reported, exposure to antigens other than related drugs may lead to specific IgE to drugs, so that hypersensitivity reactions will occur with the first exposure to drugs [33]. At present this mechanism has not been verified in oxaliplatin, but it does provide possible theoretical support for oxaliplatin in patients with allergy history so that such patients need to be highly vigilant against hypersensitivity reactions' occurrence if they infused oxaliplatin. Moreover, platinum exposure history differs from allergy history, patients with which experienced at least one exposure to platinum drugs, who may be stimulated with specific platinum IgE. Therefore, when they are exposed to oxaliplatin again, specific IgE activation leads to local or systemic hypersensitivity reactions. Sohn et al. [27] consider that in such patients hypersensitivity reactions are more likely to happen in an earlier chemotherapy cycle and show more severe symptoms, who require more cautious clinical management. In addition, some patients with exposure to oxaliplatin were medicated with oxaliplatin again after platinum free interval have increasing cumulative dose of oxaliplatin. Although patient's immune response weakened during platinum free interval, IgE will still be activated rapidly $[17,22]$. Sohn et al. found that platinum-free interval of patients in oxaliplatin hypersensitivity group was significantly longer than that of patients in non-hypersensitivity group (21.6 vs. 16.8 months, $P=0.007$ ), but the mechanism underlying longer platinum-free interval promoting hypersensitivity reactions is still unclear [27]. Therefore, for such patients with platinum-free interval, clinicians should evaluate risks of reintroduction and adopt premedication or desensitization to reduce the possibility of hypersensitivity reactions in patients.

Several studies report that premedication with steroids (e.g., dexamethasone, prednisone, methylprednisone) and antihistamines (e.g., ranitidine, diphenhydramine, promethazine) can reduce the occurrence of oxaliplatin hypersensitivity reactions to some extent $[19,34-36]$. Shen et al. enrolled 139 colorectal cancer patients receiving oxaliplatin treatment and investigated that among 14 patients occurring hypersensitivity reactions, 3 of 5 patients receiving premedication with steroids and antihistamines successfully rechallenged oxaliplatin regimen, and premedication with dexamethasone was a potential risk factor of oxaliplatin hypersensitivity reactions [19]. Analogously, in order to assess certain rechallenge protocol targeting at patients with previous hypersensitivity reactions, Wu found 15 patients who experienced hypersensitivity reactions to oxaliplatin and underwent a rechallenge protocol containing premedication with dexamethasone, chlorpheniramine, ranitidine and all of them did not encounter another hypersensitivity reaction throughout the course of oxaliplatin treatment cycle [34]. A retrospective study by Lee et al. evaluated the efficacy of premedication for controlling oxaliplatin-related hypersensitivity reactions in patients with gastrointestinal malignancy, and of the 134 patients who were administered premedication with chlorpheniramine and hydrocortisone (totally 175 patients exhibiting hypersensitivity reactions to oxaliplatin), $71.6 \%$ had complete or partial prevention of hypersensitivity reactions after premedication [35]. Moreover, Lee et al. found that the success rate of completing oxaliplatin administration by premedication decreased as the severity of hypersensitivity reactions increased [35]. 
Sakaeda et al. reached a similar conclusion through data mining of the FDA Adverse Event Reporting System that dexamethasone administration effectively reduced oxaliplatin-induced mild hypersensitivity reactions, but had less impact on severe and lethal hypersensitivity reactions, while the effects of diphenhydramine to hypersensitivity reactions were examined no signals [36]. A prospective study by Yoshita et al. concluded that simultaneous infusion of oxaliplatin and dexamethasone increased $\mathrm{pH}$ of the infusion solution, thereby inhibiting the release of histamine and reducing the occurrence of hypersensitivity reactions [37]. Some studies have further concluded that higher pretreatment dose of steroids and antihistamines can lead to lower incidence of oxaliplatin hypersensitivity reactions. A retrospective cohort study by Kidera et al. reported that increased doses of dexamethasone and antihistamines significantly reduced incidence of oxaliplatin hypersensitivity reactions [38]. In our meta-analysis, Yamauchi et al. and Kim et al. believed that dexamethasone doses less than 12 and $20 \mathrm{mg}$, respectively, were risk factors for oxaliplatin hypersensitivity reactions [26, 29]. However, this premedication method does not work for all patients. Ohta et al. thought that dexamethasone premedication had nothing to do with oxaliplatin hypersensitivity [21]. In the study by Brandi et al., six patients were administrated with dexamethasone premedication to reintroduce oxaliplatin, five of which still went through the same hypersensitivity reaction [39]. Many researchers discovered that quite a number of patients with oxaliplatin hypersensitivity reactions failed to rechallenge oxaliplatin-based chemotherapy even though they received premedication with steroids or antihistamines [40-42]. Currently, the selection and dosage of premedication drugs for oxaliplatin still lacks guidelines or high-level evidences to give standardized recommendations, so considerable work needs to be invested in the future to explore the impact of different premedication methods on oxaliplatin hypersensitivity reactions. In cases where the instructions and guidelines have not clearly indicated, clinicians generally choose premedication proposals based on personal experiences so that more attention should be paid on whether patients have hypersensitivity reactions even though with premedication.

This meta-analysis finds that age has nothing to do with oxaliplatin hypersensitivity reactions, but after excluding one study a positive conclusion is reached that younger patients are more likely to develop oxaliplatin hypersensitivity reactions. Heterogeneity among these studies attributes to the study by Yamauchi et al., which almost drew the opposite conclusion that patients with hypersensitivity reactions to oxaliplatin were 3.1 years elder than those without hypersensitivity [29]. However, this study contained only 62 patients, whose small sample size led to high specificity and affected the accuracy of overall analysis. Previous studies have reported that infants, teenagers and the elderly were risk factors of anaphylaxis [43], but the mechanism as to how age influences oxaliplatin hypersensitivity is not yet known and, therefore, further exploration is required to verify cause and effect. Another risk factor with high heterogeneity, gender, is also found to have no relation with oxaliplatin hypersensitivity reactions in our meta-analysis. Identically, with excluding one study, heterogeneity obviously reduced and women was concerned as a risk factor of oxaliplatin hypersensitivity. Some researchers believe that women are more likely to develop drug hypersensitivity reactions [32, 43], the mechanism underlying which may ascribe to hormones [23], while the study by Okayama et al. which produced heterogeneity of gender indicated that men were more likely to occur oxaliplatin hypersensitivity reaction [24]. Based on the findings above, whether gender has a significant effect on oxaliplatin hypersensitivity reactions still needs to be further explored.

The following are the potential limitations in this study: (1) all cross-sectional studies are included with lack of case-control studies and cohort studies. Evidence level of literature keeps not high enough that may cause certain potential bias and lack of persuasiveness with the conclusion to some extent; (2) some of the risk factors analyzed involve less researches and thereby the pooled results are not reliable enough that more data are required to increase the stability of the evidence; (3) this study has a wider geographical variation with most patients included belong to Asians, of which two studies' populations are from the United States and France, so certain publication bias exists. The individual differences in oxaliplatin hypersensitivity reactions actually remain, so big gap among the ethnicity of study population may exacerbate the clinical heterogeneity; (4) in this study only a risk factor with not less than three studies were analyzed. The remaining factors such as palliative chemotherapy, cumulative dose, eosinophils, albumin, etc. cannot be counted due to lack of data, on which some researches hold affirmative thoughts indeed with no explanation that they are irrelevant with oxaliplatin hypersensitivity reactions; (5) this study is a review of the current evidence without analyzing individual data. Based on the results, we assume that premedication is a point that is worth further exploration, so as the next step we are going to study the effect of different premedication regimens on oxaliplatin hypersensitivity in real-world settings.

In summary, our meta-analysis shows that platinum exposure history, allergy history and long platinum-free interval are important risk factors of oxaliplatin hypersensitivity reactions and high dexamethasone premedication dose is a protective factor of oxaliplatin hypersensitivity. The danger of hypersensitivity to oxaliplatin should not be underestimated This study may help identify patients with risk factors and conduct clinical monitoring and management so as not to affect patients' chemotherapy process and life quality. 
Additional prospective studies with larger sample size and high-level evidence are needed to further investigate risk factors associated with oxaliplatin hypersensitivity reactions.

Author contributions LZ, HL conceived and coordinated the study, designed, performed and analyzed the experiments, wrote the paper. QD, XY, SY, XL and QZ carried out the data collection, data analysis, and revised the paper. All authors reviewed the results and approved the final version of the manuscript.

Funding QD was supported by Key Innovative Team of Shanghai TopLevel University Capacity Building in Clinical Pharmacy and Regulatory Science at Shanghai Medical College, Fudan University (Shanghai Municipal Education Commission, HJW-R-2019-66-19). QD was supported by Shanghai "Rising Stars of Medical Talent" Youth Development Program Youth Medical Talents Clinical Pharmacist Program [SHWRS(2020)_087]. QZ was supported by "Clinical Research Plan of SHDC" [SHDC2020CR3085B].

Availability of data and material No additional data are available.

Code availability Not applicable.

\section{Declarations}

Conflicts of interest The authors declare that they have no conflict of interest.

Ethics approval Not applicable.

Consent to participate Not applicable.

Consent for publication Not applicable.

Open Access This article is licensed under a Creative Commons Attribution 4.0 International License, which permits use, sharing, adaptation, distribution and reproduction in any medium or format, as long as you give appropriate credit to the original author(s) and the source, provide a link to the Creative Commons licence, and indicate if changes were made. The images or other third party material in this article are included in the article's Creative Commons licence, unless indicated otherwise in a credit line to the material. If material is not included in the article's Creative Commons licence and your intended use is not permitted by statutory regulation or exceeds the permitted use, you will need to obtain permission directly from the copyright holder. To view a copy of this licence, visit http://creativecommons.org/licenses/by/4.0/.

\section{References}

1. Bray F, Ferlay J, Soerjomataram I et al (2018) Global cancer statistics 2018: GLOBOCAN estimates of incidence and mortality worldwide for 36 cancers in 185 countries. CA Cancer J Clin 68:394-424. https://doi.org/10.3322/caac.21492

2. Grothey A, Goldberg RM (2004) A review of oxaliplatin and its clinical use in colorectal cancer. Expert Opin Pharmacother 5:2159-2170. https://doi.org/10.1517/14656566.5.10.2159

3. Rogers BB, Cuddahy T, Briscella C et al (2019) Oxaliplatin: detection and management of hypersensitivity reactions. Clin $\mathrm{J}$ Oncol Nurs 23:68-75. https://doi.org/10.1188/19.CJON.68-75
4. Shepherd GM (2003) Hypersensitivity reactions to chemotherapeutic drugs. Clin Rev Allergy Immunol 24:253-262. https://doi. org/10.1385/CRIAI:24:3:253

5. Toki MI, Saif MW, Syrigos KN (2014) Hypersensitivity reactions associated with oxaliplatin and their clinical management. Expert Opin Drug Saf 13:1545-1554. https://doi.org/10.1517/14740338. 2014.963551

6. Saif MW (2006) Hypersensitivity reactions associated with oxaliplatin. Expert Opin Drug Saf 5:687-694. https://doi.org/10.1517/ 14740338.5.5.687

7. Bano N, Najam R, Qazi F et al (2016) Clinical features of oxaliplatin induced hypersensitivity reactions and therapeutic approaches. Asian Pac J Cancer Prev 17:1637-1641. https://doi. org/10.7314/apjcp.2016.17.4.1637

8. Aroldi F, Prochilo T, Bertocchi P et al (2015) Oxaliplatininduced hypersensitivity reaction: underlying mechanisms and management. J Chemother 27:63-66. https://doi.org/10.1179/ 1973947814Y.0000000204

9. Makrilia N, Syrigou E, Kaklamanos I et al (2010) Hypersensitivity reactions associated with platinum antineoplastic agents: a systematic review. Met Based Drugs. https://doi.org/10.1155/ 2010/207084

10. Stroup DF, Berlin JA, Morton SC et al (2000) Meta-analysis of observational studies in epidemiology: a proposal for reporting. Meta-analysis Of Observational Studies in Epidemiology (MOOSE) group. JAMA 283:2008-2012. https://doi.org/10. 1001/jama.283.15.2008

11. Rostom A, Dubé C, Cranney A et al (2004) Celiac Disease. Rockville (MD): Agency for Healthcare Research and Quality (US); 2004 Sep (Evidence Reports/Technology Assessments, No. 104.) Appendix D. Quality Assessment Forms. https:// www.ncbi.nlm.nih.gov/books/NBK35156/

12. Ouyang M, Liao T, Lu Y et al (2019) Laparoscopic versus open surgery in lateral lymph node dissection for advanced rectal cancer: a meta-analysis. Gastroenterol Res Pract 2019:7689082. https://doi.org/10.1155/2019/7689082

13. Hu J, Dong Y, Chen X et al (2015) Prevalence of suicide attempts among Chinese adolescents: a meta-analysis of crosssectional studies. Compr Psychiatry 61:78-89. https://doi.org/ 10.1016/j.comppsych.2015.05.001

14. Lau J, Ioannidis JP, Schmid CH (1997) Quantitative synthesis in systematic reviews. Ann Intern Med 127:820-826. https:// doi.org/10.7326/0003-4819-127-9-199711010-00008

15. Higgins JP, Thompson SG, Deeks JJ et al (2003) Measuring inconsistency in meta-analyses. BMJ 327:557-560. https://doi. org/10.1136/bmj.327.7414.557

16. Begg CB, Mazumdar M (1994) Operating characteristics of a rank correlation test for publication bias. Biometrics 50:1088-1101

17. Li XQ, Yang QL, He GZ et al (2018) Clinical analysis of hypersensitivity reactions to oxaliplatin. Chin J Clin Oncol 45:12681271. https://doi.org/10.3969/j.issn.1000-8179.2018.24.055

18. Du CX, Hong RX, Chen Z et al (2014) Clinical characteristics of oxaliplatin-induced hypersensitivity reactions. Chin J Clin Oncol Rehabil 21:194-197. https://doi.org/10.13455/j.cnki.cjcor.2014. 02.21

19. Shen XP, Liu X, Qian H et al (2013) Clinical analysis of hypersensitivity reaction to oxaliplatin in patients with colorectal cancer. J Chin Oncol 19:623-627. https://doi.org/10.11735/j.issn.1671170X.2013.08.B008

20. Zhu QY, Wang YY, Liu XL et al (2017) Clinical analysis of hypersensitivity reactions to oxaliplatin in patients with colorectal cancer. Anti Tumor Pharmacy 7:509-512. https://doi.org/10.3969/j. issn.2095-1264.2017.04.26

21. Ohta H, Hayashi T, Murai S et al (2017) Comparison between hypersensitivity reactions to cycles of modified FOLFOX6 and 
XELOX therapies in patients with colorectal cancer. Cancer Chemother Pharmacol 79:1021-1029. https://doi.org/10.1007/ s00280-017-3294-9

22. Mori Y, Nishimura T, Kitano T et al (2010) Oxaliplatin-free interval as a risk factor for hypersensitivity reaction among colorectal cancer patients treated with FOLFOX. Oncology 79:136-143. https://doi.org/10.1159/000320613

23. Kim BH, Bradley T, Tai J et al (2009) Hypersensitivity to oxaliplatin: an investigation of incidence and risk factors, and literature review. Oncology 76:231-238. https://doi.org/10.1159/000205263

24. Okayama T, Ishikawa T, Sugatani K et al (2015) Hypersensitivity reactions to oxaliplatin: identifying the risk factors and judging the efficacy of a desensitization protocol. Clin Ther 37:12591269. https://doi.org/10.1016/j.clinthera.2015.03.012

25. Parel M, Ranchon F, Nosbaum A et al (2014) Hypersensitivity to oxaliplatin: clinical features and risk factors. BMC Pharmacol Toxicol 15:1. https://doi.org/10.1186/2050-6511-15-1

26. Kim MY, Kang SY, Lee SY et al (2012) Hypersensitivity reactions to oxaliplatin: clinical features and risk factors in Koreans. Asian Pac J Cancer Prev 13:1209-1215. https://doi.org/10.7314/apjcp. 2012.13.4.1209

27. Sohn KH, Kang DY, Kim JY et al (2018) Incidence and risk of oxaliplatin-induced hypersensitivity in patients with asymptomatic prior exposure: a prospective observational study. J Allergy Clin Immunol Pract 6(1642-1648):e1642. https://doi.org/10. 1016/j.jaip.2017.12.026

28. Shibata Y, Ariyama H, Baba E et al (2009) Oxaliplatininduced allergic reaction in patients with colorectal cancer in Japan. Int J Clin Oncol 14:397-401. https://doi.org/10.1007/ s10147-009-0883-6

29. Yamauchi H, Goto T, Takayoshi $\mathrm{K}$ et al (2015) A retrospective analysis of the risk factors for allergic reactions induced by the administration of oxaliplatin. Eur J Cancer Care (Engl) 24:111116. https://doi.org/10.1111/ecc.12156

30. Seki K, Senzaki K, Tsuduki Y et al (2011) Risk factors for oxaliplatin-induced hypersensitivity reactions in Japanese patients with advanced colorectal cancer. Int J Med Sci 8:210-215. https://doi. org/10.7150/ijms.8.210

31. Caiado J, Picard M (2014) Diagnostic tools for hypersensitivity to platinum drugs and taxanes: skin testing, specific IgE, and mast cell/basophil mediators. Curr Allergy Asthma Rep 14:451. https:// doi.org/10.1007/s11882-014-0451-7

32. Waheed A, Hill T, Dhawan N (2016) Drug allergy. Prim Care 43:393-400. https://doi.org/10.1016/j.pop.2016.04.005

33. Wheatley LM, Plaut M, Schwaninger JM et al (2015) Report from the National Institute of Allergy and Infectious Diseases workshop on drug allergy. J Allergy Clin Immunol 136(262-271):e262. https://doi.org/10.1016/j.jaci.2015.05.027
34. Wu HL (2019) Retrospective evaluation of a rechallenge protocol in patients experiencing hypersensitivity reactions with prior chemotherapy in a tertiary hospital. J Oncol Pharm Pract 25:1388-1395. https://doi.org/10.1177/1078155218796190

35. Lee SY, Kang HR, Song WJ et al (2014) Overcoming oxaliplatin hypersensitivity: different strategies are needed according to the severity and previous exposure. Cancer Chemother Pharmacol 73:1021-1029. https://doi.org/10.1007/s00280-014-2437-5

36. Sakaeda T, Kadoyama K, Yabuuchi H et al (2011) Platinum agentinduced hypersensitivity reactions: data mining of the public version of the FDA adverse event reporting system, AERS. Int J Med Sci 8:332-338. https://doi.org/10.7150/ijms.8.332

37. Yoshida Y, Hirata K, Matsuoka H et al (2015) A single-arm Phase II validation study of preventing oxaliplatin-induced hypersensitivity reactions by dexamethasone: the AVOID trial. Drug Des Devel Ther 9:6067-6073. https://doi.org/10.2147/DDDT.S94901

38. Kidera Y, Satoh T, Ueda S et al (2011) High-dose dexamethasone plus antihistamine prevents colorectal cancer patients treated with modified FOLFOX6 from hypersensitivity reactions induced by oxaliplatin. Int J Clin Oncol 16:244-249. https://doi.org/10.1007/ s10147-010-0170-6

39. Brandi G, Pantaleo MA, Galli C et al (2003) Hypersensitivity reactions related to oxaliplatin (OHP). Br J Cancer 89:477-481. https://doi.org/10.1038/sj.bjc.6601155

40. Syrigou EI, Karapanagiotou EM, Alamara CV et al (2009) Hypersensitivity reactions to oxaliplatin: a retrospective study and the development of a desensitization protocol. Clin Colorectal Cancer 8:106-109. https://doi.org/10.3816/CCC.2009.n.017

41. Polyzos A, Tsavaris N, Gogas H et al (2009) Clinical features of hypersensitivity reactions to oxaliplatin: a 10-year experience. Oncology 76:36-41. https://doi.org/10.1159/000178163

42. Lenz G, Hacker UT, Kern W et al (2003) Adverse reactions to oxaliplatin: a retrospective study of 25 patients treated in one institution. Anticancer Drugs 14:731-733. https://doi.org/10. 1097/00001813-200310000-00007

43. Simons FE, Ardusso LR, Dimov V et al (2013) World Allergy Organization Anaphylaxis Guidelines: 2013 update of the evidence base. Int Arch Allergy Immunol 162:193-204. https://doi. org/10.1159/000354543

Publisher's Note Springer Nature remains neutral with regard to jurisdictional claims in published maps and institutional affiliations. 